\title{
Usefulness of preoperative thoracic paravertebral block in postoperative pain management after renal resection surgery
}

\author{
Copik M. Białka S. Daszkiewicz A. Misiołek H.
}

\section{School of Medicine with Division of Dentistry in Zabrze \\ Medical University of Silesia \\ Department of Anesthesia, Intensive Care and Emergency Medicine Zabrze Poland}

\section{Background and goal}

Thoracic paravertebral block (ThPVB) combined with general anesthesia is commonly used in thoracic surgery. It provides effective analgesia, reduces surgical stress response and incidence of chronic postoperative pain compared to solely used general anaesthesia. The goal of the study was to assess the usefulness of ThPVB in postoperative pain management after renal resection surgery.

\section{Material and methods}

Patients ASA I-III scheduled for elective renal resection surgery were randomly assigned to two groups $(n=58)$. PVB group $(n=27)$ received preoperative ThPVB with $0,5 \%$ bupivacaine followed by general anaesthesia. GEN group $(n=31)$ received standard general anaesthesia. Both groups were treated postoperatively with oxycodone IV PCA (patient controlled analgesia) combined with non-opioid analgesics as rescue drugs. We recorded pain severity in VAS, oxycodone requirement in time points, total oxycodone requirement, and sedation level through the first $48 \mathrm{~h}$. We measured opioid related adverse events 24 and 48 h postoperatively and patients satisfaction $48 \mathrm{~h}$ postoperatively. Data were analysed by using unpaired Student's t-test, Mann-Whitney U-test and $\chi 2$ test. Analysis of the changes over time was performed using parametrical variance analysis. Results are presented as mean $\pm \mathrm{sd}$, median or percentage. $\mathrm{P}<0.05$ was considered statistically significant.

\section{Results}

Patients given ThPVB needed $39 \%$ less IV oxycodone in the first $48 \mathrm{~h}$, had a longer latency to the first opioid dose, and less pain at rest in VAS scale through first $24 \mathrm{~h}$ than the control(p < 0.01 ). They had less opioid related adverse events after 24 and $48 \mathrm{~h}$ and were less sedated until $12 \mathrm{~h}$ postoperatively. Patients in PVB group also had higher satisfaction scores after $48 \mathrm{~h}$ postoperatively compared to control group.

\section{Conclusion}

In our study preoperative ThPVB was an effective part of multimodal analgesia regimen in reducing opioid consumption and pain intensity.Methods and drugs used in both groups can be considered safe with no serious AE recorded. Majority of non-serious $\mathrm{AE}$ was opioid related adverse events.Patients in ThPVB group reported increased satisfaction compared to the control group.
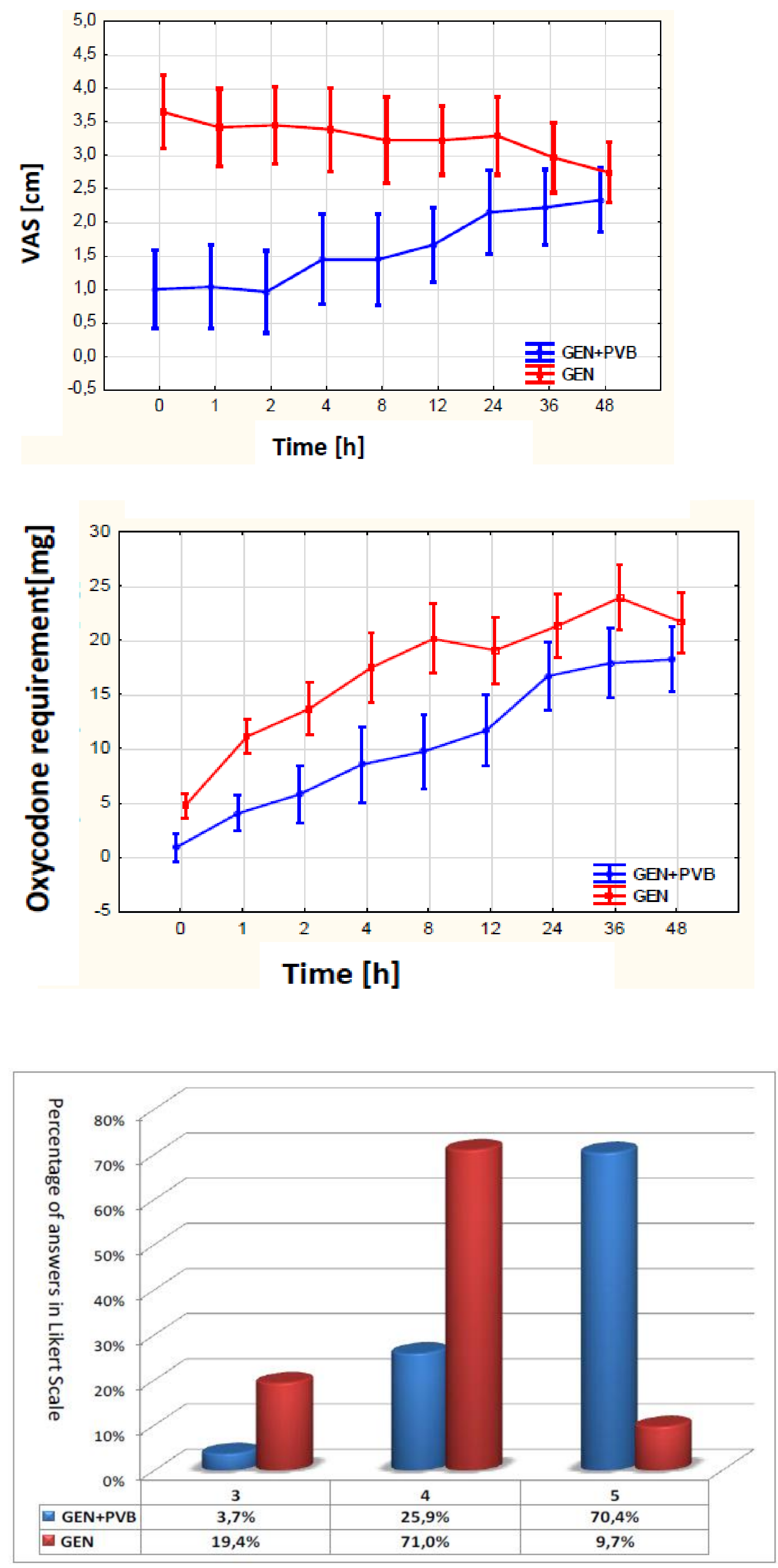